East African Medical Journal Vol. 85 No. 5 May 2008

UNUSUAL PRESENTATION OF IDIOPATHIC RETROPERITONEAL FIBROSIS: CASE REPORT

N. Twahirwa, MBChB, Radiology Resident and J. Rees, MBChB (Edin), FRCR, FRCP, Professor and Director, PGME,

Department of Radiology, Aga Khan University Hospital, Nairobi, P.O. Box 30270-00100, Nairobi, Kenya

Request for reprints to: Dr. N. Twahirwa, Aga Khan University Hospital, Nairobi, P.O. Box 30270-00100, Nairobi, Kenya

\title{
UNUSUAL PRESENTATION OF IDIOPATHIC RETROPERITONEAL FIBROSIS: CASE REPORT
}

\author{
N. TWAHIRWA and J. REES
}

\begin{abstract}
SUMMARY
Idiopathic retroperitoneal fibrosis (IRF) is an uncommon entity described as progressive proliferation of connective tissues leading to a fibrous plaque-like lesions that encases the aorta and inferior vena cava inferior to the level of the renal arteries. Mass forming retroperitoneal fibrosis is rare. We present a rare case of a unilateral focal retroperitoneal mass simulating a tumour encasing the middle third of the left ureter, with involvement of the ipsilateral scrotum.
\end{abstract}

\section{INTRODUCTION}

Idiopathic retroperitoneal fibrosis (IRF) is an uncommon entity described as progressive proliferation of connective tissues leading to a fibrous plaque-like lesions that encase the aorta and inferior vena cava inferior to the level of the renal arteries. Mass forming retroperitoneal fibrosis is rare.

\section{CASE REPORT}

A37year old male presented at Aga Khan University Hospital accident and emergency department in July 2007 with a two-week history of intermittent sharp left flank pain with no radiation or aggravating/relieving factors. He denied any episode of fever, dysuria, frequency, urgency or haematuria. There was no known allergy or chronic medication history. A right inguinal lymphnode was excised at nine years, which was benign on histology. Examination revealed tender and rigid left flank muscles. Urolithiasis was queried and CT KUB requested.

CT KUB and abdomen: Unenhanced CT KUB (Figures 1 and 2) demonstrated a left iso-attenuating unilateral focal retroperitoneal mass encasing the middle third of the ureter and extending inferiorly along the psoas muscle (measuring crania-caudal $14.2 \mathrm{~cm}$, anteroposterior $3.4 \mathrm{~cm}$ and transverse $5.6 \mathrm{~cm}$ ) with proximal hydroureter and perinephric stranding. The peritoneum was otherwise clear and no calculus was seen.

On standard CT abdominal examination (Figures 3 and 4) the mass showed enhancement equivalent to the adjacent psoas muscle. The left perinephric stranding and hydroureter seen are due to the obstructive effect of the mass. A differential diagnoses included lymphoma, retroperitoneal fibrosis and sarcoma.

Biopsy and histology: The patient underwent CT guided biopsy and histological examination (Figure 5) confirmed the features consistent with retroperitoneal fibrosis. On clinical review he was found to have a left hydrocele that was confirmed on ultrasound. Both testes were normal (Figures 6 and 7). The patient thereafter had double J stenting and Jabouleys procedure for the ureteral obstruction and hydrocele respectively. Post operatively he was started on tamoxifen 20mg BD. 


\section{Figures 1 and 2}

Axial sections of CT KUB demonstrating left retroperitoneal iso-attenuating mass abutting the ipsilateral psoas muscle with associated hydroureter and perinephric stranding
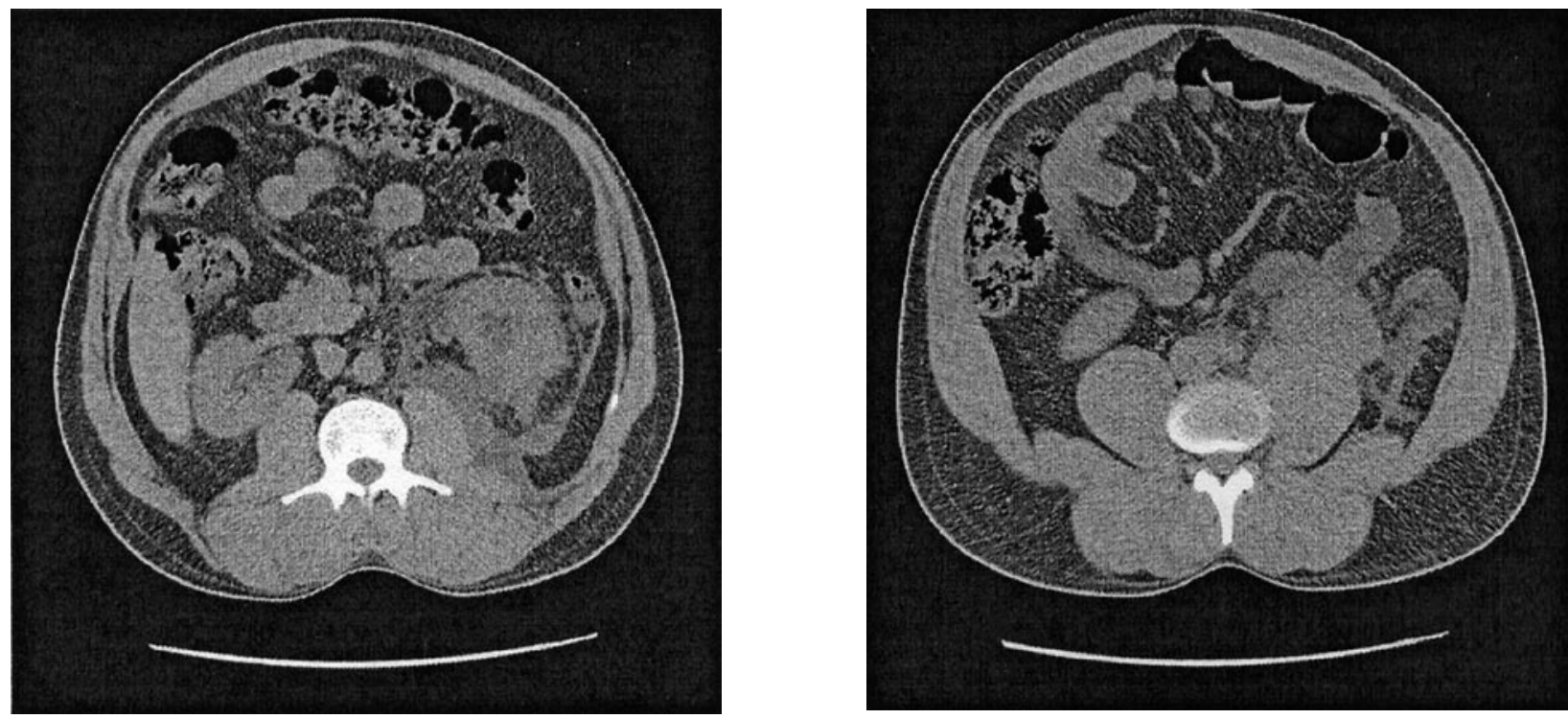

Figures 3 and 4

Axial and coronal enhanced CT abdomen re-demonstrated left periureteral mass that has enhancement equivalent to the adjacent psoas muscle
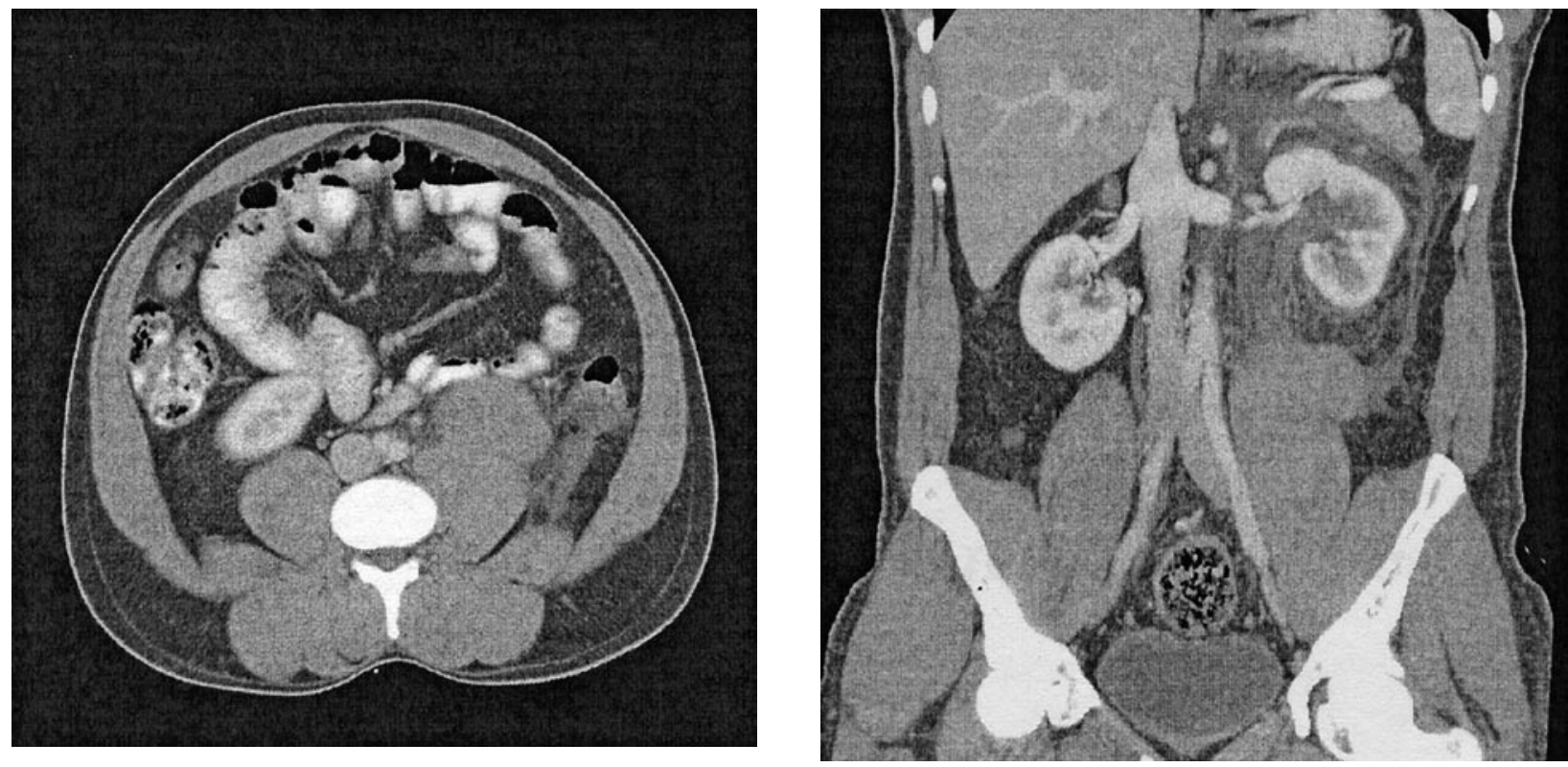


\section{Figure 5}

Histology micrograph shows dense fibrous and fatty connective tissue infiltrated by a mixed inflammatory cell infiltrate comprising of occasional neutrophils, eosinophils and a predominace of lymphocytes with dispersed plasma cells and histiocytes

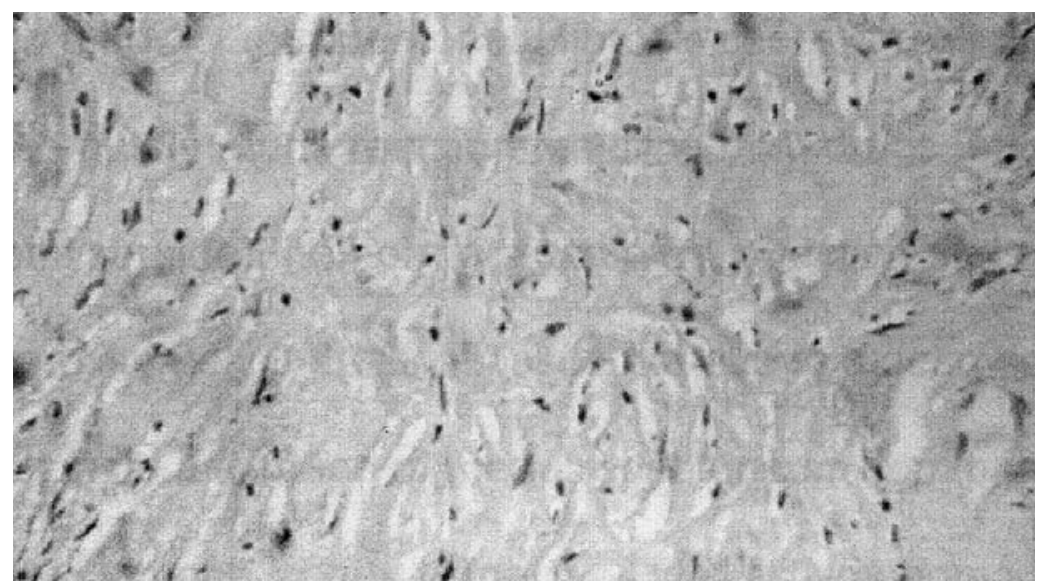

Figures 6 and 7

Bilateral testicular ultrasound, demonstrating left sided hydrocele and normal testicular parenchymal

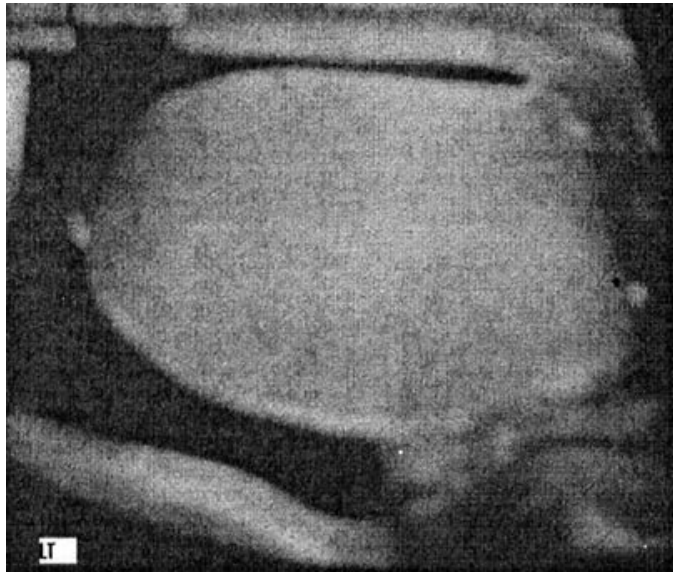

\section{DISCUSSION}

Retroperitoneal fibrosis is a rare disease entity with an estimated incidence of 1:200 000 population. Albarran reported the first documented case in 1905, and Ormond established the disease entity in 1948(1), explaining why it's also known as "Ormond's syndrome".

Two thirds of retroperitoneal fibrosis cases are idiopathic. The remainder are due to an autoimmune reaction resulting from drugs (such as amphetamines and methysergide), haemorrhage, surgery, infection and abdominal aortic aneurysm. Approximately 8-10\% of cases are due to a desmoplastic reaction caused by malignant infiltration of the retroperitoneum from primary tumours of the breast, lung, stomach, colon,

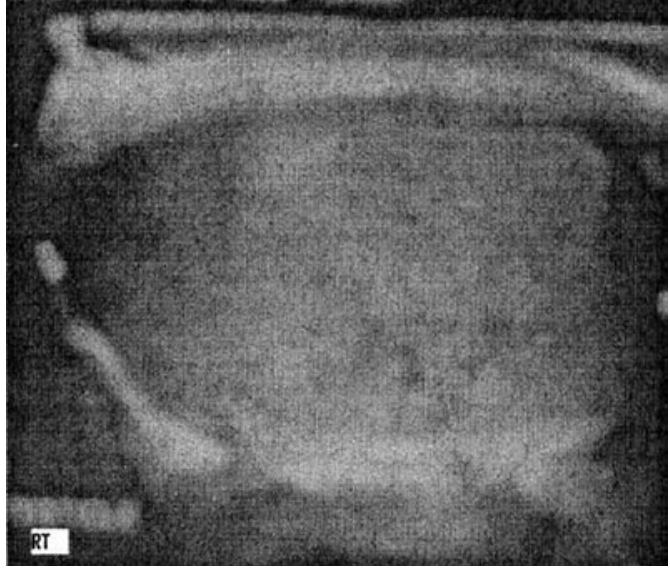

kidneys as well as lymphoma and sarcoma (2). Systemic fibrosing diseases that have been associated with retroperitoneal fibrosis include fibrous pseudotumour of the orbit, Riedel's thyroiditis, sclerosing cholangitis and mediastinal fibrosis (3).

Tuberculosis has also been associated with retroperitoneal fibrosis (2). Pathologically, IRF manifests as progressive inflammation and fibrous proliferation of connective tissue over the anterior surface of the lower lumbar vertebrae, below the renal arteries, encasing the inferior vena cava and aorta (4). Up to $15 \%$ have extra retroperitoneal foci (5). It characteristically causes medial deviation of the middle third of the ureter and rarely invades the ureteric wall, but if it does the mucosa is in most cases spared. Hydronephrosis is due to the absence 
of peristalsis, the ureters being fixed open by the disease process. The duodenum, biliary tract and pancreas are rarely involved (1).

Histological examination usually demonstrates fibrous overgrowth with accompanying inflammatory infiltrate of lymphocytes, plasma cells, and neutrophils, suggesting inflammatory rather than neoplastic disease.

Mass forming Idiopathic IRF, as in our case is very rare. To our knowledge only 18 cases including ours have been reported (Table 1). When seen it can be located any where in the retroperitoneum from the diaphragm to the pelvis (4). Figure 8 shows another example identified by the authors in 2002, demonstrating a left perinephric mass that was proven as retroperitoneal fibrosis and improved on tamoxifen. In these cases it is very difficult to distinguish the resultant mass from a retroperitoneal malignancy, especially lymphoma and retroperitoneal sarcoma.

The clinical presentation of IRF is non-specific consisting of dull, poorly localized back or flank pain, weight loss, anorexia, nausea or vomiting and malaise. There is significant morbidity due to progressive renal failure resulting from ureteral entrapment $(1,4)$.
Intravenous urography may show the classic triad of delayed excretion of contrast material with unilateral $(20 \%)$ or bilateral hydronephrosis, medial deviation of the middle third of the ureter and tapered narrowing of the ureters at L4/5 level (1). Ultrasound may show a relatively echo-poor retroperitoneal mass and hydronephrosis (6).

\section{Figure 8}

Coronal CT showing a left perinephric mass and associated reduced size and enhancement of ipsilateral kidney

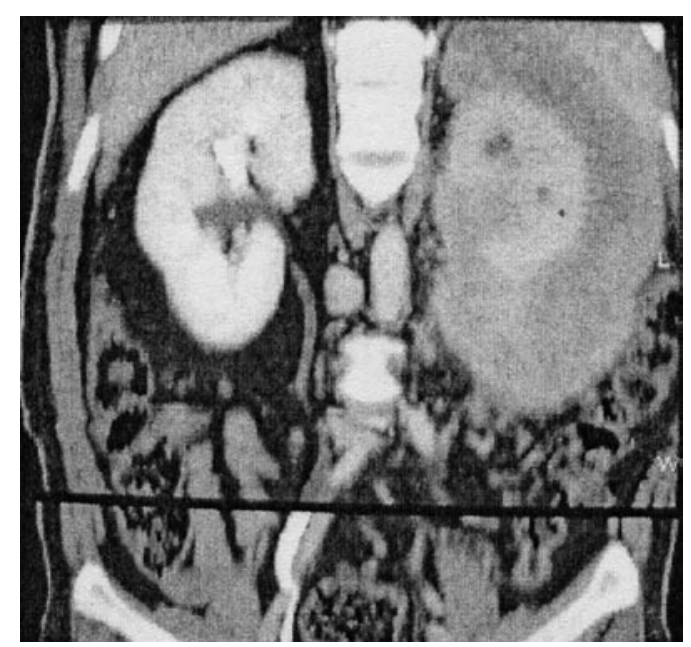

Table 1

Reported cases tumour forming idiopathic retroperitoneal fibrosis (1, 3, 4 and 5)

\begin{tabular}{llccll}
\hline Year & Author & Age & Gender & Location & Tumour size \\
\hline 1978 & Chi & 42 & M & Lt. kidney & $17 \times 4$ \\
1985 & Arai & 69 & F & Pelvis & $5 \times 5$ \\
1988 & Stovall & 34 & F & Pelvis & $15 \times 12$ \\
1991 & Muurata & 36 & F & Rt. ureter & $6 \times 4$ \\
1996 & Touge & 54 & F & Pelvis & $4 \times 4$ \\
1997 & Oh & 30 & M & Rt. kidney & $19 \times 15$ \\
1998 & Kuwabara & 71 & M & Bilat. kidneys & $4 \times 4,3 \times 3$ \\
1998 & Tekeyama & 30 & F & Pelvis & $5 \times 3$ \\
1998 & Haciyanli & 60 & M & Pelvis & $6 \times 4$ \\
1999 & Koie & 59 & M & Pelvis & $3 \times 3$ \\
1999 & Dejaco & 46 & M & Hepatic hilum & $2 \times 2$ \\
2000 & Nakase & 55 & M & Lt. kidney & $6 \times 6$ \\
2000 & Wong & 3 & F & Rt. ureter & $2 \times 2$ \\
2003 & Tsutomu & 76 & F & Rt. adrenal & $8 \times 7$ \\
2003 & Buyl & 65 & M & Lt. ureter & - \\
2004 & Warakaulle & 73 & M & Retrocrural & $4 \times 5$ \\
2006 & Das & 18 & F & Rt. ureter & - \\
2007 & Present case & 37 & M & Lt. ureter & $14.2 \times 5.6 \times 3.4$ \\
\hline
\end{tabular}


CT and magnetic resonance imaging (MRI) are the modalities of choice for imaging RPF. On $\mathrm{CT}$, retroperitoneal fibrosis appears as plaquelike, soft tissue lesion of variable thickness that envelops the aorta and IVC between the renal hila and sacral promontory, and extends laterally to entrap the ureters, resulting in variable degrees of hydronephrosis. It does not cause anterior displacement of the aorta and when this occurs, lymphoma or other widespread malignancy should be considered (1). The plaque is isodense to muscle on unenhanced CT. The early, active vascular stage shows significant enhancement, whereas the avascular later stage enhances very little. Calcification is rare and has been reported in only two cases of mass forming IRF $(1,2)$.

MRI has the advantages of superior soft tissue contrast and multiplanar imaging. Haem sequences may also be used to detect retroperitoneal haemorrhage, as it is a potential differential diagnosis.

RPF is generally of low signal intensity on $T_{1}$ weighted image. On $\mathrm{T}_{2}$ weighted image, there is high signal intensity due to high cellularity and fluid content in the early active phase, while in the late fibrous phase signal intensity is low due to increased deposition of fibrous tissue (1). IRF usually enhances after intravenous gadolinium administration, except when in a quiescent stage (7). Imaging appearances of IRF can be mimicked by other conditions such as malignant lymphadenopathy, haemorrhage, infection and amyloidosis (3).

MR urography can be performed in the same setting whilst avoiding potentially harmful iodinated contrast media in patients with impaired renal function.

The other unique aspect in our patient was the associated ipsilateral hydrocele. Scrotal involvement has been described due to entrapped gonadal vessels and lymphatics that cause scrotal oedema (8).
Steroid therapy is effective for treating early stage IRF, as are other drugs such as cyclosporine, cytotoxic agents and tamoxifen. Ureterolysis or stents can be performed for ureteral obstruction (3).

In conclusion, a focal and unilateral mass forming idiopathic retroperitoneal fibrosis is rare, however it should be kept in mind as a differential diagnosis of retroperitoneal masses and image guided biopsy must be performed in such cases for definite diagnosis.

\section{REFERENCES}

1. Das, C.J., Sharma, R., Jain, T.P., et al. Unusual appearance of retroperitoneal fibrosis simulating a tumour. Brit. J. Radiol. 2006; 79: e137-e139.

2. Scully, R.E., Mark, E.J. and McNeely W.F. Case records of Massachusetts General Hospital. Weekly clinicopathological exercises. N. Engl. J. Med. 1995; 332: 174-179.

3. Warakulle, D.R., Prematilleke, I. and Moore, N.R. Retroperitoneal fibrosis mimicking retrocrural lymphadenopathy. Clin. Radiol. 2004; 59: 2923. (CrossRefl/Medline).

4. Takashima, T., Onoda, N., Ishikawa, T., et al. Tumor forming idiopathic retroperitoneal fibrosis: Report of a case. Surg. Today. 2004; 34: 374-378. (CrossRefl/ Medline).

5. Buyl, L., Oosterlinck, W., Verstraete, K., et al. An unusual case of unilateral periureteral retroperitoneal fibrosis. Clin. Radiol. 2003; 58: 4924. (CrossRefl/Med me).

6. Amis, E.S. Retroperitoneal fibrosis. A.J.R. Amer. J. Roentgenol. 1991; 157: 321-329.

7. Lee, J.K.,Hiken, J.N. and Semelka R.C. Retroperitoneum: Computed body tomography with MRI correlation. Philadelphia, PA: Lippincott Raven, 1998; 1070.

8. Biyani, C.S., et al. Retroperitoneal fibrosis. Emedicine last updated November 30, 2006. 